TENDENCIAS

Revista de la Facultad de Ciencias

Económicas y Administrativas.

Universidad de Nariño

ISSN-E 2539-0554

Vol. XXII No. 2 - 2do Semestre 2021

Julio-Diciembre - Páginas 26-56

\title{
EVALUACIÓN DE POLÍTICAS PÚBLICAS DE CAPITAL HUMANO EN CIENCIA Y TECNOLOGÍA EN MÉXICO, 2012-2018
}

EVALUATION OF PUBLIC POLICIES ON HUMAN CAPITAL IN SCIENCE AND

TECHNOLOGY IN MEXICO, 2012-2018

\section{AVALIAÇÃO DE POLÍTICAS PÚBLICAS DE CAPITAL HUMANO EM CIÊNCIA E TECNOLOGIA NO MÉXICO, 2012-2018}

\author{
Darío Figueroa; Natalí Basilico
}

Doctorando en Ciencias Económicas, Universidad Autónoma de Baja California, México. Maestro en Estudios del Desarrollo Global, Universidad Autónoma de Baja California, México. Magíster en Dirección de Empresas y Negocios, Universidad Austral, Argentina. ORCiD: 0000-0002-13954423. E-mail: figueroa.dario@uabc.edu.mx, México.

Doctorando en Ciencias Económicas, Universidad Autónoma de Baja California, México. Maestra en Ciencias Económicas, Universidad Autónoma de Baja California, México. ORCiD: 0000-00034082-0727. E-mail: nbasilico@uabc.edu.mx, México.

Recibido: 3 de enero de 2021

Aprobado: 10 de marzo de 2021

DOI: https://doi.org/10.22267/rtend.212202.167

\section{Resumen}

El bajo desarrollo del capital humano fue señalado como uno de los obstáculos del progreso de México, de acuerdo con un diagnóstico inicial del gobierno de Enrique Peña Nieto. La presente investigación se propone evaluar el desempeño de políticas públicas para la formación y fortalecimiento del capital humano en ciencia y tecnología, implementadas por el Gobierno 
Federal de México entre 2012 y 2018. Para ello, se analiza el logro de los objetivos propuestos en documentos oficiales de planeación, a través del contraste de indicadores oficiales y propios con los resultados obtenidos. Se reconoce el cumplimiento de parte de los objetivos de la política pública, como el incremento de los miembros del Sistema Nacional de Investigadores (SNI) y los avances en las acciones para favorecer la participación de las mujeres en el sistema científico. Sin embargo, no fue alcanzado el objetivo de promoción del capital humano en áreas prioritarias ni fue posible reconocer con claridad un incremento en el nivel de los investigadores del SNI, ya que se hallaron aspectos favorables y desfavorables.

Palabras clave: competitividad; desarrollo de recursos humanos; desarrollo económico; investigación y desarrollo; gasto público; planificación del desarrollo.

JEL: H11; H52; I28; J24; O21; O38

\begin{abstract}
The low development of human capital was pointed out as one of the obstacles to progress in Mexico, according to an initial diagnosis by the government of Enrique Peña Nieto. The aim of this study is to evaluate the performance of public policies implemented by the Mexican Federal Government on the training and strengthening of human capital in science and technology between 2012 and 2018. For this, the achievement of the objectives proposed in official planning documents is analyzed through the contrast between official and own indicators and the outcomes achieved by government policies. Results of the study show compliance with part of public policy objectives due to the increase in the number of members of the National System of Researchers (SNI), and progress in actions to favor women's participation in the scientific system. However, the objective of promoting human capital in priority areas was not achieved, and it was not possible to clearly recognize an increase in the level of SNI researchers since favorable and unfavorable aspects were found.
\end{abstract}

Keywords: competitiveness; human resources development; economic development; research and development; public expenditure; development planning.

JEL: H11; H52; I28; J24; O21; O38 


\section{Resumo}

O baixo desenvolvimento do capital humano foi apontado como um dos obstáculos ao progresso do México, segundo um diagnóstico inicial do governo de Enrique Peña Nieto. Assim, este estudo objetiva avaliar o desempenho das políticas públicas de formação e fortalecimento do capital humano em ciência e tecnologia, implementadas pelo Governo Federal do México entre 2012 e 2018. Para isso, analisa-se o cumprimento dos objetivos propostos nos documentos oficiais de planejamento, por meio do contraste de indicadores oficiais e próprios com os resultados alcançados pelas políticas governamentais. A pesquisa demonstrou o cumprimento de uma parte dos objetivos das políticas públicas, devido à ampliação do quadro de integrantes do Sistema Nacional de Pesquisadores (SNI) assim como ao avanço nas ações que favorecem a participação das mulheres no sistema científico. No entanto, não foi alcançado o objetivo de promoção do capital humano em áreas prioritárias, e não foi possível reconhecer claramente um aumento do nível de investigadores do SNI, uma vez que foram encontrados aspectos favoráveis e desfavoráveis.

Palavras-chave: competitividade; desenvolvimento de recursos humanos; desenvolvimento económico; pesquisa e desenvolvimento; despesa pública; planejamento do desenvolvimento.

JEL: H11; H52; I28; J24; O21; O38

\section{Introducción}

Tal como sucede en muchos países de la región, México ha enfrentado problemas de competitividad en distintas dimensiones que le han impedido posicionarse como una potencia global. La llegada del presidente Enrique Peña Nieto a finales de 2012 - al igual que lo que ocurre con cada cambio de administración en el Poder Ejecutivo Nacional - representó una nueva oportunidad para repensar los objetivos y acciones que impulsen la prosperidad del país.

Precisamente, ese fue el espíritu del Plan Nacional de Desarrollo 2013-2018 (PND13-18), un documento estratégico elaborado por el Gobierno Federal de México para comunicar los objetivos que se buscarían alcanzar durante el sexenio. Uno de los aspectos allí identificados como obstáculos para el progreso del país fue el bajo nivel de desarrollo del capital humano, entendido como el conjunto de conocimientos, habilidades, competencias y atributos de las personas que 
permiten alcanzar bienestar personal, social y económico (Keeley, 2007). El diagnóstico plasmado en el PND13-18 reflejaba un sistema de educación deficiente y una vinculación inadecuada entre los sectores educativo, empresarial y social (Poder Legislativo, 2013).

En este sentido, la gestión de Peña Nieto se propuso promover políticas que acercaran los conocimientos y las habilidades brindados en las escuelas a las demandas del mercado, así como alcanzar una mayor y más efectiva inversión en ciencia y tecnología para incrementar el valor agregado y transformar a México en una sociedad basada en el conocimiento (Poder Legislativo, 2013). El logro de estos desafíos resulta crucial y debe ser un imperativo para el sector público (Licona y Pérez, 2018), ya sea para mejorar el desempeño económico, tanto a nivel nacional como regional (Organización para la Cooperación y el Desarrollo Económicos [OCDE], 2010) como para aumentar, simultáneamente, la eficiencia y la igualdad (Destinobles, 2006).

El objetivo del presente trabajo consiste en evaluar el desempeño de políticas públicas implementadas en México durante el sexenio 2012-2018 en materia de formación y fortalecimiento de capital humano en ámbitos científicos y tecnológicos, para conocer el grado de cumplimiento de los objetivos planteados en los documentos oficiales de planificación e identificar fortalezas y aspectos a mejorar.

El documento se estructura de la siguiente manera: la sección 1 revisa el concepto de capital humano, su relación con el desarrollo y su abordaje desde las políticas públicas en México; la sección 2 detalla la metodología utilizada; la sección 3 expone los resultados; y, finalmente, se presentan las conclusiones y reflexiones del trabajo.

\section{El impulso al capital humano altamente especializado como medio para el desarrollo en México}

\section{El capital humano: una llave para el desarrollo}

El concepto de capital humano fue introducido por Theodore Schultz (1959) para referirse a las inversiones en las personas que permiten generar flujos de ingresos adicionales. Las nociones de Schultz fueron ampliamente desarrolladas por Gary Stanley Becker (1964), quien presentó a la 
educación y al entrenamiento como inversiones que los individuos eligen realizar de manera racional con el objetivo de incrementar su productividad e ingresos.

A grandes rasgos, se suelen identificar tres tipos de procesos de acumulación de capital humano a lo largo de la vida de las personas, de acuerdo con el momento o lugar en el que se generan: el que se adquiere en el hogar; el que se acumula por experiencia; y el que se obtiene por medio de la educación formal (Destinobles, 2006). El capital humano tiene como efecto incrementar la productividad económica de cada individuo; por consiguiente, a nivel agregado, a medida que aumenta el capital humano de un país, mayor se espera que sea su nivel de producción (Destinobles, 2006).

Entre las diferentes acepciones del capital humano, la educación formal ha sido de las variables más estudiadas en la literatura debido a la posibilidad de capturar, medir, explicar y comparar el comportamiento de distintas economías mediante la utilización de modelos matemáticos. A partir de la segunda mitad del siglo XX, la teoría del Capital Humano logró un gran protagonismo entre los académicos. Múltiples investigaciones posicionaron al capital humano como uno de los factores más importantes para el crecimiento económico (Romer, 1986; Lucas, 1988; Barro, 1989; Barro y Sala-i-Martin, 1995; Barro, 2001) debido a su relación positiva y significativa con la productividad (Romer, 1989; Mankiw et al., 1992; Benhabib y Spiegel, 1994).

Bajo este último enfoque, las posibilidades de un territorio para incrementar su competitividad y ofrecer un mayor bienestar a la población están altamente condicionadas por el nivel de desarrollo tecnológico, el que a su vez depende de las políticas públicas de ciencia y tecnología, la orientación de la educación al desarrollo científico y tecnológico y la vinculación y coherencia de su sistema científico-tecnológico (Cabello y Ortiz, 2013).

\section{Un rezago persistente}

América Latina cuenta con viejos pero severos problemas en relación con el desarrollo tecnológico. Si bien la política de industrialización por sustitución de importaciones, auge entre las décadas de 1930 y 1960, arrojó resultados positivos, no logró impulsar el desarrollo de innovaciones ni evitar el rezago competitivo de los sectores productivos, fenómeno que Fajnzylber 
(1983) denominó como "la industrialización trunca de América Latina”. Con la disrupción de la fase de globalización iniciada en la segunda mitad del siglo pasado, las barreras nacionales se redujeron drásticamente y las diferencias en el nivel de desarrollo tecnológico entre los países del centro y de la periferia se hicieron más evidentes. Los beneficios del mayor intercambio solo pudieron ser aprovechados por los países que se encontraban en condiciones de competir en los mercados internacionales; en tanto, los gobiernos latinoamericanos debieron recurrir a un proteccionismo frívolo, con altas tasas de ganancias para empresarios poco innovadores (Fajnzylber, 1983).

De este modo, la región fue quedando inmersa en un fuerte proceso de desindustrialización, que se profundizó a partir de la década de 1990. Desde entonces, la mayor parte de las exportaciones latinoamericanas se componen, principalmente, por productos primarios en el sur del continente, y por bienes ensamblados de "alta tecnología" pero con bajo valor agregado en México y en el Caribe (Vazquez, 2017).

Precisamente, México es un claro ejemplo de este problema. Más allá de haber celebrado diversos tratados de libre comercio con distintos países y regiones durante las últimas décadas, y de haber acatado las indicaciones de la Organización Mundial del Comercio, el Fondo Monetario Internacional y el Banco Mundial, su economía continúa estancada. Los principales aspectos de este lento crecimiento se relacionan con un proceso de "desindustrialización precoz", caracterizado por la consolidación de un modelo industrial maquilador, de baja inversión y bajo valor agregado y de alta dependencia de las exportaciones a Estados Unidos (Calderón-Villarreal y HernándezBielma, 2016).

Al mismo tiempo, la incapacidad de la industria de absorber capital humano calificado, sumado a la hipertrofia de los servicios y la pérdida de competitividad de las exportaciones manufactureras de los sectores no maquiladores, profundizaron la pérdida de dinamismo de la industria y la emigración de mexicanos, fundamentalmente hacia Estados Unidos y Canadá (Calderón-Villarreal y Hernández-Bielma, 2016). 


\section{La intervención del Estado y la necesidad de evaluar sus acciones}

Es evidente que el "desafío del conocimiento" que Fajnzylber ya advertía en 1992 (incrementar los esfuerzos en educación, investigación científica y modernización tecnológica) no solo no está resuelto, sino que adquiere cada vez más el carácter de urgente, debido al advenimiento de la sociedad del conocimiento.

El sector público juega un rol protagónico en dicho desafío: identificar los nichos en los cuales es posible obtener alguna ventaja competitiva y elaborar políticas públicas de largo plazo para resolver problemas que, por su relevancia para importantes sectores de la sociedad, no son factibles de enfrentarse eficazmente desde el ámbito privado (Cardozo, 2013).

El Estado, como cualquier otro agente, se enfrenta al problema de la elección, es decir, debe decidir dónde aplicar sus recursos finitos, en un contexto de necesidades colectivas ilimitadas. Es por ello que, una vez sorteado dicho problema y realizada la ejecución de la acción gubernamental, es importante identificar en qué medida se lograron los objetivos que se perseguían a través de la utilización de los recursos públicos. Es allí donde la evaluación de las políticas públicas aparece como un instrumento indispensable para conocer y mejorar las acciones desarrolladas por los gobiernos (Roth, 2009), por medio de la recolección, verificación e interpretación de la información sobre la ejecución de las políticas y programas (Majone, 1997).

Mediante la evaluación, se completa una secuencia, descripta por Jones (1970), que se compone por la identificación de un problema, la formulación de alternativas de solución, la elección de una de ellas y su posterior implementación, y que puede dar lugar tanto a un reajuste de la política como a su eliminación (Roth, 2009).

\section{El rol del Estado en la ciencia, la tecnología y la innovación en México}

La política de ciencia, tecnología e innovación en México se estructura a través de un amplio entramado institucional que va de lo general a lo particular, tal como se presenta en la Figura 1. En primer lugar, la Constitución Política (1917, p. fracción V, artículo 3ro) establece que “el Estado promoverá y atenderá todos los tipos y modalidades educativos (...) necesarios para el 
desarrollo de la nación, apoyará la investigación científica y tecnológica, y alentará el fortalecimiento y difusión de nuestra cultura".

\section{Figura 1}

Entramado institucional de las acciones en ciencia, tecnología e innovación en México

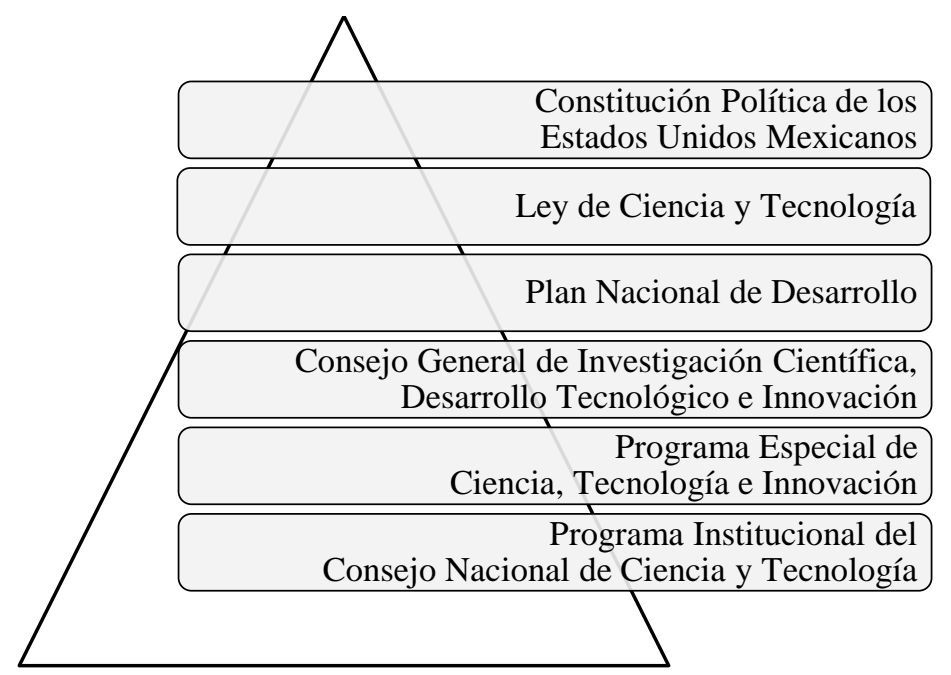

Fuente: elaboración propia.

Por su parte, la Ley de Ciencia y Tecnología (LCyT) (2002) menciona los medios y mecanismos que el Estado debe garantizar para asegurar el cumplimiento de la carta magna. En tanto, el Plan Nacional de Desarrollo (PND), elaborado por el presidente de la República y aprobado por el Congreso de la Unión, establece los objetivos y estrategias nacionales que servirán de base para los programas sectoriales, especiales, institucionales y regionales durante una administración.

Al Consejo General de Investigación Científica, Desarrollo Tecnológico e Innovación, presidido por el presidente de la República, le corresponde la definición de prioridades y criterios para la asignación del gasto público federal en ciencia, tecnología e innovación. El Programa Especial de Ciencia, Tecnología e Innovación (PECiTI), consagrado en la LCyT y elaborado por el Consejo Nacional de Ciencia y Tecnología (CONACyT), detalla los medios para alcanzar los objetivos propuestos en el PND. Por último, el Programa Institucional del CONACyT establece un conjunto de objetivos, estrategias y líneas de acción que deben contribuir al logro de las metas nacionales en materia de ciencia, tecnología e innovación. 
Las piezas del entramado institucional antes descripto integran el Sistema Nacional de Ciencia, Tecnología e Innovación, cuya estructura se representa en la Figura 2. Este sistema es coordinado por el CONACyT y se compone por los instrumentos de gobierno, política pública y planeación y por un conjunto diverso y heterogéneo de actores, entre los que se encuentran: el sector público, en sus tres niveles; el sector académico y de investigación; y empresas con actividades en materia científica y tecnológica (CONACyT, 2014a).

\section{Figura 2}

Composición del Sistema Nacional de Ciencia, Tecnología e Innovación de México

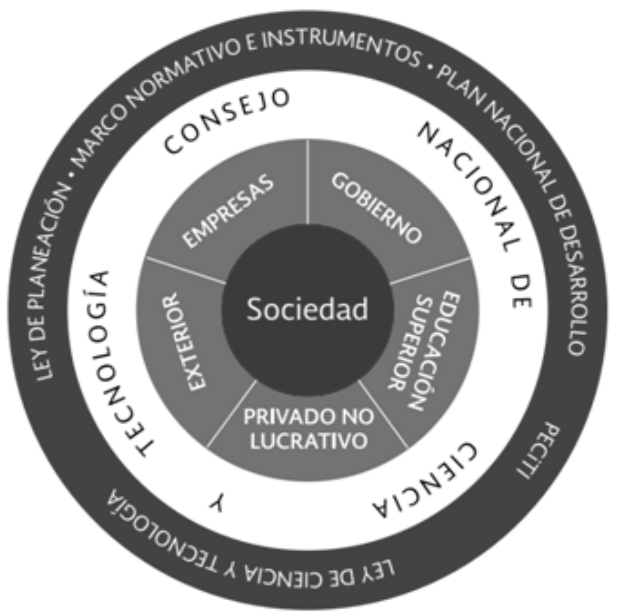

Fuente: CONACyT (2014a).

\section{La formación y fortalecimiento del capital humano en ciencia y tecnología en México durante el sexenio 2012-2018}

Las acciones tendientes al desarrollo del capital humano en ciencia y tecnología durante el período 2012-2018 se plasmaron en el PND13-18 y en el PECiTI14-18. El primero, se estructuró a partir de un objetivo general o visión, que sirvió de marco para las cinco metas nacionales y las tres estrategias transversales, tal como se muestra en la Tabla 1.

Las políticas de ciencia y tecnología formaron parte de la Meta Nacional III, la que a su vez se integró por cinco objetivos particulares, cuyo detalle se presenta en la Tabla 2. 
Tabla 1

Esquema del Plan Nacional de Desarrollo 2013-2018

\begin{tabular}{llll}
\hline \multicolumn{1}{c}{ Objetivo General } & \multicolumn{2}{c}{ Metas Nacionales } & \multicolumn{1}{c}{ Estrategias Transversales } \\
\hline & I & México en Paz & i) Democratizar la productividad \\
Llevar a México a & II & México Incluyente & \\
su máximo & III & México con Educación de Calidad & ii) Gobierno cercano y moderno \\
potencial & IV & México Próspero & \\
& & México con Responsabilidad & iii) Perspectiva de género \\
& V & Global & \\
\hline
\end{tabular}

Fuente: elaboración propia, en base al PND13-18 de México (Poder Legislativo, 2013).

\section{Tabla 2}

Objetivos particulares de la Meta Nacional III del Plan Nacional de Desarrollo 2013-2018

\begin{tabular}{crl}
\hline Meta Nacional & \multicolumn{1}{c}{ Objetivo Particular } \\
\hline & 3.1 & $\begin{array}{l}\text { Desarrollar el potencial humano de los mexicanos con educación de } \\
\text { calidad. }\end{array}$ \\
& $3.2 \begin{array}{l}\text { Garantizar la inclusión y la equidad en el Sistema Educativo. } \\
\text { México con } \\
\text { III educación } \\
\text { de calidad }\end{array}$ & $3.3 \begin{array}{l}\text { Ampliar el acceso a la cultura como un medio para la formación integral } \\
\text { de los ciudadanos. }\end{array}$ \\
& $3.4 \begin{array}{l}\text { Promover el deporte de manera incluyente para fomentar una cultura de } \\
\text { salud. } \\
\text { Hacer del desarrollo científico, tecnológico y la innovación pilares para } \\
\text { el progreso económico y social sostenible. }\end{array}$ \\
\hline
\end{tabular}

Fuente: elaboración propia, en base al PND 13-18 de México (Poder Legislativo, 2013).

El Objetivo Particular 3.5 del PND13-18 se convirtió en el objetivo general del PECiTI14-18. Esta alineación buscó la coordinación de las dependencias y entidades de la Administración Pública Federal que realizan actividades en materia de ciencia, tecnología e innovación. Entre los distintos objetivos particulares del PECiTI14-18, el 3.5.2 denominado "contribuir a la formación y fortalecimiento del capital humano de alto nivel", fue el que abordó el desarrollo de capital humano en ciencia y tecnología. Éste se compuso, a su vez, de una serie de estrategias y líneas de acción que, posteriormente, se vincularon con los distintos programas sectoriales (Tabla 3). 


\section{Tabla 3}

Planificación de políticas públicas en formación y fortalecimiento del capital humano en ciencia y tecnología en México, 2012-2018

\begin{tabular}{|c|c|c|c|c|c|}
\hline \multicolumn{6}{|c|}{ PECiTI14-18 } \\
\hline $\begin{array}{l}\text { Objetivo } \\
\text { General }\end{array}$ & $\begin{array}{l}\text { Objetivo } \\
\text { Particular }\end{array}$ & Estrategia & $\begin{array}{l}\text { Línea de } \\
\text { acción }\end{array}$ & $\begin{array}{l}\text { Objetivo del } \\
\text { Programa } \\
\text { Educación }\end{array}$ & $\begin{array}{c}\text { Línea de Acción } \\
\text { del Programa }\end{array}$ \\
\hline \multirow{3}{*}{$\begin{array}{l}3.5 . \\
\text { Hacer del } \\
\text { desarrollo } \\
\text { científico, } \\
\text { tecnológico } \\
\text { y la } \\
\text { innovación } \\
\text { pilares para } \\
\text { el progreso } \\
\text { económico } \\
\text { y social } \\
\text { sostenible }\end{array}$} & \multirow{3}{*}{$\begin{array}{l}3.5 .2 \text {. } \\
\text { Contribuir a la } \\
\text { formación y } \\
\text { fortalecimiento } \\
\text { del capital } \\
\text { humano de } \\
\text { alto nivel }\end{array}$} & $\begin{array}{l}2.1 . \\
\text { Consolidar la } \\
\text { masa crítica } \\
\text { de } \\
\text { investigadores } \\
\text { para generar } \\
\text { investigación } \\
\text { científica y } \\
\text { desarrollo } \\
\text { tecnológico } \\
\text { de alto nivel }\end{array}$ & $\begin{array}{l}\text { 2.1.1. } \\
\text { Incrementar } \\
\text { el número } \\
\text { de } \\
\text { científicos y } \\
\text { tecnólogos } \\
\text { del SNI en } \\
\text { prioridades } \\
\text { del sector de } \\
\text { ciencia, } \\
\text { tecnología e } \\
\text { innovación }\end{array}$ & $\begin{array}{l}\text { 2. Fortalecer la } \\
\text { calidad y } \\
\text { pertinencia de } \\
\text { la educación } \\
\text { media superior, } \\
\text { superior y } \\
\text { formación para } \\
\text { el trabajo, a fin } \\
\text { de que } \\
\text { contribuyan al } \\
\text { desarrollo de } \\
\text { México }\end{array}$ & $\begin{array}{l}2.4 .7 . \\
\text { Incrementar el } \\
\text { número y el nivel } \\
\text { de los } \\
\text { investigadores de } \\
\text { las instituciones } \\
\text { de educación } \\
\text { superior en el } \\
\text { SNI }\end{array}$ \\
\hline & & $\begin{array}{l}2.2 . \\
\text { Generar los } \\
\text { mecanismos } \\
\text { que }\end{array}$ & $\begin{array}{l}2.2 .2 . \\
\text { Incrementar } \\
\text { el número }\end{array}$ & $\begin{array}{l}\text { 6. Impulsar la } \\
\text { educación } \\
\text { científica y } \\
\text { tecnológica } \\
\text { como elemento }\end{array}$ & $\begin{array}{l}6.3 .2 . \\
\text { Incrementar el } \\
\text { número de } \\
\text { becarias y } \\
\text { becarios de } \\
\text { posgrado en } \\
\text { ciencias e } \\
\text { ingeniería }\end{array}$ \\
\hline & & $\begin{array}{l}\text { conectar la } \\
\text { oferta y la } \\
\text { demanda de } \\
\text { recursos } \\
\text { humanos de } \\
\text { alto nivel }\end{array}$ & $\begin{array}{l}\text { posgrado } \\
\text { otorgadas } \\
\text { por el } \\
\text { Gobierno } \\
\text { Federal }\end{array}$ & $\begin{array}{l}\text { indispensable } \\
\text { para la } \\
\text { transformación } \\
\text { de México en } \\
\text { una sociedad } \\
\text { del } \\
\text { conocimiento }\end{array}$ & $\begin{array}{l}\text { 6.3.3. Impulsar } \\
\text { acciones } \\
\text { afirmativas en } \\
\text { becas de } \\
\text { posgrados para la } \\
\text { integración de } \\
\text { mujeres en } \\
\text { carreras } \\
\text { científicas y } \\
\text { técnicas }\end{array}$ \\
\hline
\end{tabular}

Fuente: elaboración propia, en base al CONACyT (2014a). 


\section{Metodología}

Se realizó una evaluación ex post contrastando los documentos oficiales de planificación estratégica (Figura 3) con los informes de gestión y la información disponible en el Sistema Integrado de Información sobre Investigación Científica, Desarrollo Tecnológico e Innovación, del Gobierno Federal de México. Esto permitió valorar el conjunto de efectos, directos o indirectos, previstos o no, que la intervención realizada pudo haber provocado en el problema que la originó (Cardozo, 2013), utilizando un conjunto de indicadores para comprobar si hubo o no un acercamiento progresivo a las metas propuestas. Si bien el sexenio se extendió desde finales del 2012 hasta finales del 2018, la evaluación se llevó a cabo para el período 2014-2018, tomando como línea de base el año 2013, tal como se contempla en el PECiTI14-18.

\section{Figura 3}

Mapa de evaluación de las políticas de formación y fortalecimiento del capital humano en ciencia, tecnología e innovación en México, 2012-2018

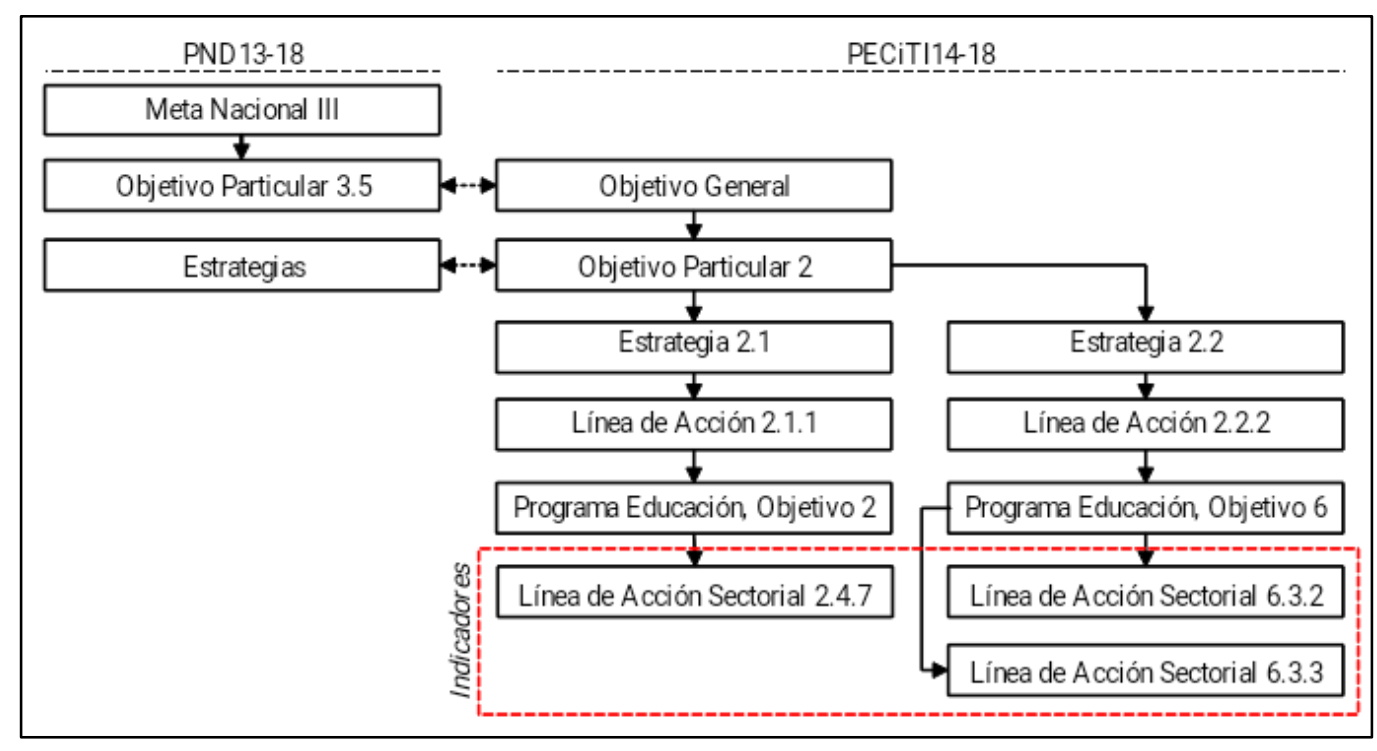

Fuente: elaboración propia.

Si bien el PECiTI14-18 elaboró indicadores para poder evaluar el cumplimiento de sus objetivos particulares, no los asoció a las Líneas de Acción Sectorial (LAS). Por lo tanto, a los fines del presente trabajo, dicha asociación fue inferida en función la definición y contenido de cada uno de ellos. Sin embargo, y siguiendo este razonamiento, no fue posible vincular ningún indicador oficial 
a la LAS 6.3.3. En este sentido, se diseñó un conjunto de indicadores propios que, si bien no permitieron evaluar (debido a la imposibilidad de fijar metas), abonaron al análisis. Los indicadores utilizados se presentan en la Tabla 4 y se explican en el Anexo A.

\section{Tabla 4}

Indicadores para evaluar y analizar las líneas de acción del programa sectorial Educación del Objetivo Particular 2 del PECiTI14-18

\begin{tabular}{|c|c|c|}
\hline Línea de acción sectorial & Número y nombre del indicador & Fuente \\
\hline \multirow{4}{*}{$\begin{array}{l}\text { 2.4.7. Incrementar el } \\
\text { número y el nivel de los } \\
\text { investigadores de las } \\
\text { instituciones de educación } \\
\text { superior en el SNI }\end{array}$} & $\begin{array}{l}\text { 1. Porcentaje de investigadores por cada } 1.000 \\
\text { personas de la PEA ocupada }\end{array}$ & PECiTI14-18 \\
\hline & $\begin{array}{l}\text { 2. Artículos científicos publicados por cada } \\
\text { millón de habitantes }\end{array}$ & PECiTI14-18 \\
\hline & $\begin{array}{l}\text { 3. Porcentaje de variación de la cantidad de } \\
\text { investigadores del SNI }\end{array}$ & $\begin{array}{l}\text { Elaboración } \\
\text { propia }\end{array}$ \\
\hline & $\begin{array}{l}\text { 4. Porcentaje de investigadores en los niveles II } \\
\text { y III del SNI }\end{array}$ & $\begin{array}{l}\text { Elaboración } \\
\text { propia }\end{array}$ \\
\hline \multirow{3}{*}{$\begin{array}{l}6.3 .2 \text { Incrementar el } \\
\text { número de becarias y } \\
\text { becarios de posgrado en } \\
\text { ciencias e ingeniería }\end{array}$} & $\begin{array}{l}\text { 5. Porcentaje de graduados de doctorado en } \\
\text { ciencias e ingenierías respecto al total de } \\
\text { graduados de doctorado }\end{array}$ & PECiTI14-18 \\
\hline & $\begin{array}{l}\text { 6. Porcentaje de becas del CONACyT en } \\
\text { ciencias e ingenierías }\end{array}$ & $\begin{array}{l}\text { Elaboración } \\
\text { propia }\end{array}$ \\
\hline & $\begin{array}{l}\text { 7. Porcentaje de investigadores del SNI en } \\
\text { ciencias e ingenierías }\end{array}$ & $\begin{array}{l}\text { Elaboración } \\
\text { propia }\end{array}$ \\
\hline \multirow{4}{*}{$\begin{array}{l}\text { 6.3.3 Impulsar acciones } \\
\text { afirmativas en becas de } \\
\text { posgrados para la } \\
\text { integración de mujeres en } \\
\text { carreras científicas y } \\
\text { técnicas }\end{array}$} & $\begin{array}{l}\text { 8. Porcentaje de Becas Nacionales adjudicadas } \\
\text { por el CONACyT a mujeres }\end{array}$ & $\begin{array}{l}\text { Elaboración } \\
\text { propia }\end{array}$ \\
\hline & $\begin{array}{l}\text { 9. Porcentaje de Becas Nacionales adjudicadas } \\
\text { por el CONACyT a mujeres, por área del } \\
\text { conocimiento }\end{array}$ & $\begin{array}{l}\text { Elaboración } \\
\text { propia }\end{array}$ \\
\hline & 10. Porcentaje de mujeres en el SNI & $\begin{array}{l}\text { Elaboración } \\
\text { propia }\end{array}$ \\
\hline & $\begin{array}{l}\text { 11. Porcentaje de mujeres en el SNI, por área } \\
\text { del conocimiento }\end{array}$ & $\begin{array}{l}\text { Elaboración } \\
\text { propia }\end{array}$ \\
\hline
\end{tabular}

Fuente: elaboración propia. 


\section{Resultados}

\section{Evaluación de la Línea de Acción Sectorial 2.4.7}

Para el indicador "Porcentaje de investigadores por cada 1.000 personas de la PEA ocupada", el PECiTI14-18 estimó un valor de base en 2013 de 0,94 y metas anuales hasta el 2018 (Tabla 5). Posteriormente, el CONACyT (2017) corrigió el valor de base (a 0,58), aunque no los valores de las metas, que quedaron asociados a la base inicial. Por ello, si bien el indicador mostró un desempeño positivo entre 2014 y 2018, no fue posible determinar si los resultados alcanzaron o no las expectativas, ya que las metas anuales pudieron haber quedado sobrevaluadas.

\section{Tabla 5}

Investigadores por cada 1.000 personas de la PEA ocupada

\begin{tabular}{lccccccc}
\hline Variable & Versión & 2013 & 2014 & 2015 & 2016 & 2017 & 2018 \\
\hline \multirow{2}{*}{ Línea base } & Inicial & 0,94 & & & & & \\
& Corregida & 0,58 & & & & & \\
Meta & & & 0,99 & 1,05 & 1,10 & 1,15 & 1,20 \\
Resultado & & & 0,61 & 0,62 & 0,64 & 0,68 & 0,70 \\
\hline Diferencia & & & $\mathrm{n} / \mathrm{c}$ & $\mathrm{n} / \mathrm{c}$ & $\mathrm{n} / \mathrm{c}$ & $\mathrm{n} / \mathrm{c}$ & $\mathrm{n} / \mathrm{c}$ \\
\hline
\end{tabular}

Fuente: elaboración propia, en base al CONACyT (2015, 2016, 2017, 2018, 2019a).

La Tabla 6 se corresponde con el análisis del indicador "Artículos científicos publicados por cada millón de habitantes". Si bien se observa una caída en 2015, desde entonces el indicador inició una trayectoria de crecimiento y se ubicó, a partir de 2017, por encima de las metas. Esto se refleja en el incremento de la participación de México en la producción mundial de artículos durante el período bajo análisis, la cual pasó de 5,8 por cada 1.000 artículos en 2014 a 6,7 en 2018. Además, el país logró mantener el segundo lugar en América Latina, por debajo de Brasil (CONACyT, 2014b y 2019b). Una de las posibles explicaciones sobre el comportamiento de este indicador podría radicar en el incremento del número de miembros del SNI, ya que, entre los diversos requisitos de este sistema, se encuentra la publicación de artículos para lograr el ingreso, la permanencia y la promoción. 


\section{Tabla 6}

Artículos científicos publicados por cada millón de habitantes

\begin{tabular}{lcccccc}
\hline Variable & 2013 & 2014 & 2015 & 2016 & 2017 & 2018 \\
\hline Línea base & 94,4 & & & & & \\
Meta & & 98,7 & 102,8 & 110,0 & 115,0 & 115,0 \\
Resultado & & 103,0 & 99,6 & 104,7 & 117,2 & 123,4 \\
\hline Diferencia & 4,3 & $-3,2$ & $-5,3$ & 2,2 & 8,4 \\
\hline
\end{tabular}

Fuente: elaboración propia, en base al CONACyT (2015, 2016, 2017, 2018, 2019a).

Por otro lado, la variación porcentual en la cantidad de investigadores del SNI mostró valores positivos durante todo el período (Tabla 7). El número de miembros del sistema creció de 19.747 en 2013 a 28.633 en 2018, lo que representa un aumento del 45\%. Este incremento se explica con la variación en el presupuesto destinado al SNI, que en 2018 fue un 58,8\% mayor al del 2013 (CONACyT, 2019b) y refleja el énfasis que la gestión de Peña Nieto otorgó al fortalecimiento de las capacidades del sistema científico-tecnológico en lo referente a la disponibilidad de recursos humanos.

\section{Tabla 7}

Porcentaje de variación anual de la cantidad de investigadores del SNI

\begin{tabular}{llllllll}
\hline Variable & 2013 & 2014 & 2015 & 2016 & 2017 & 2018 \\
\hline $\begin{array}{l}\text { Línea base } \\
\text { Resultado }\end{array}$ & 6,39 & & & & & \\
\hline
\end{tabular}

Fuente: elaboración propia, en base al CONACyT (s. f.).

Respecto a la composición del SNI, se observó una caída gradual en el porcentaje de investigadores en los niveles superiores (II y III) del 26,72\% en 2013 hasta el 24,24\% en 2018 (Tabla 8). Considerando el crecimiento en la cantidad de investigadores en ese período (Tabla 7), es posible inferir que la menor concentración de profesionales en los más altos niveles pudo deberse, fundamentalmente, a un mayor ingreso de nuevos investigadores en los niveles iniciales (Candidato y nivel I), en comparación con aquellos que lograron ascender a los niveles II y III. 


\section{Tabla 8}

Porcentaje de investigadores en los niveles II y III del SNI

\begin{tabular}{ccccccc}
\hline Variable & 2013 & 2014 & 2015 & 2016 & 2017 & 2018 \\
\hline Línea base & 26,72 & & & & & \\
Resultado & & 25,56 & 25,59 & 25,20 & 24,67 & 24,24 \\
\hline
\end{tabular}

Fuente: elaboración propia, en base al CONACyT (s. f.).

\section{Evaluación de la Línea de Acción Sectorial 6.3.2}

Respecto a la LAS 6.3.2, resulta importante aclarar que ni el PECiTI14-18 ni otros documentos del CONACyT mencionaron cuáles son las disciplinas comprendidas dentro el grupo de las "ciencias". Esto impide conocer con claridad cómo se compone la categoría de las "ciencias e ingenierías", objeto del presente indicador oficial. A los fines de este análisis, y en función de la clasificación disciplinar del CONACyT, se consideraron dentro de este agrupamiento a las siguientes áreas de conocimiento: I) Físico-Matemáticas y Ciencias de la Tierra; II) Biología y Química; VI) Biotecnología y Ciencias Agropecuarias; y VII) Ingenierías.

El indicador del CONACyT denominado "Porcentaje de graduados de doctorado en ciencias e ingenierías respecto al total de graduados de doctorado" (Tabla 9), no reflejó con exactitud el objetivo de la LAS bajo análisis (que se refiere a becarias y becarios, y no a graduados); sin embargo, se asoció a esta línea ya que se trata de las mismas áreas del conocimiento. Este indicador, también sufrió una corrección en su valor de base (que pasó de 53,6 a 43,7), pero no en sus metas anuales, que quedaron expresadas en función del valor original. Debido a esta situación, no pudieron conocerse las diferencias entre los resultados previstos y los alcanzados. De todos modos, dado que el valor del indicador retrocedió entre 2013 y 2018, es posible afirmar que el desempeño de la línea de acción, en función de este indicador, no fue satisfactorio.

El detalle sobre el porcentaje de nuevas Becas Nacionales del CONACyT adjudicadas para la realización de posgrados en ciencias e ingenierías (Tabla 10) demostró que la mayor parte de las postulaciones que este organismo decidió financiar se dirigieron a estas áreas del conocimiento. De hecho, su valor creció levemente entre 2015 y 2017 (únicos años con información oficial hallada), lo que refleja un comportamiento que acompaña a los objetivos de la política pública. Lo 
anterior toma mayor relevancia si se considera que el promedio de estudiantes de licenciatura egresados de disciplinas de ciencias e ingenierías entre 2011 y 2017 solo representó el 28,9\% del total (CONACyT, 2019b).

\section{Tabla 9}

Porcentaje de graduados de doctorado en ciencias e ingenierías respecto al total de graduados de doctorado

\begin{tabular}{lccccccc}
\hline \multicolumn{1}{c}{ Variable } & Versión & 2013 & 2014 & 2015 & 2016 & 2017 & 2018 \\
\hline Línea base & Inicial & 53,6 & & & & & \\
& Corregida & 43,7 & & & & & \\
Meta & & & 54,1 & 54,6 & 55.0 & 55.5 & 56,0 \\
Resultado & & & 40,3 & 38,4 & 40,1 & 39,7 & 38,9 \\
\hline Diferencia & & & $\mathrm{n} / \mathrm{c}$ & $\mathrm{n} / \mathrm{c}$ & $\mathrm{n} / \mathrm{c}$ & $\mathrm{n} / \mathrm{c}$ & $\mathrm{n} / \mathrm{c}$ \\
\hline
\end{tabular}

Fuente: elaboración propia, en base al CONACyT (2015, 2016, 2017, 2018, 2019a).

\section{Tabla 10}

Porcentaje de becas del CONACyT en ciencias e ingenierías

\begin{tabular}{lcccccc}
\hline Variable & 2013 & 2014 & 2015 & 2016 & 2017 & 2018 \\
\hline $\begin{array}{l}\text { Línea base } \\
\text { Resultado }\end{array}$ & $\mathrm{s} / \mathrm{i}$ & $\mathrm{s} / \mathrm{i}$ & 54,03 & 53,24 & 54,84 & $\mathrm{~s} / \mathrm{i}$ \\
\hline
\end{tabular}

Fuente: elaboración propia, en base al CONACyT (s. f.).

Lo mismo ocurrió con el porcentaje de investigadores del SNI en ciencias e ingenierías (Tabla 11). Si bien sus valores se ubicaron por encima del 56\% y mostraron un leve incremento entre 2016 y 2018, la escasa cantidad de observaciones impide afirmar si se trató de una consecuencia esperada de las políticas del gobierno o si fue un comportamiento eventual.

\section{Tabla 11}

Porcentaje de investigadores del SNI en ciencias e ingenierías

\begin{tabular}{lcccccc}
\hline Variable & 2013 & 2014 & 2015 & 2016 & 2017 & 2018 \\
\hline $\begin{array}{l}\text { Línea base } \\
\text { Resultado }\end{array}$ & $\mathrm{s} / \mathrm{i}$ & $\mathrm{s} / \mathrm{i}$ & $\mathrm{s} / \mathrm{i}$ & 56,78 & 57,40 & 57,14 \\
\hline
\end{tabular}

Fuente: elaboración propia, en base al CONACyT (s. f.). 
La Tabla 12 busca ampliar el análisis anterior, incorporando ahora la clasificación por niveles del SNI. Con relación a ello, se observó que el porcentaje de investigadores del nivel III en ciencias e ingenierías fue mayor al de los niveles I y II en todos los años; sin embargo, entre 2016 y 2018 (únicos años con datos), esa diferencia se redujo respecto del nivel I y se amplió con el II. Asimismo, los ingresos al nivel "Candidato" en estas disciplinas superaron, en todo momento, al promedio de los niveles I, II y III, mostrando un comportamiento positivo en relación el objetivo de la política pública. No obstante, este porcentaje cayó del 62,17\% al 57,59\% entre 2016 y 2018, lo que significa que la participación relativa de los nuevos investigadores en áreas no prioritarias se incrementó, contrario a los objetivos esperados.

\section{Tabla 12}

Porcentaje de investigadores del SNI en ciencias e ingenierías, por niveles

\begin{tabular}{lcccccc}
\hline \multicolumn{1}{c}{ Nivel } & $\begin{array}{c}2013 \\
\text { (línea base) }\end{array}$ & 2014 & 2015 & 2016 & 2017 & 2018 \\
\hline Candidato & $\mathrm{s} / \mathrm{i}$ & $\mathrm{s} / \mathrm{i}$ & $\mathrm{s} / \mathrm{i}$ & 62,17 & 60,23 & 57,59 \\
$\mathrm{I}$ & $\mathrm{s} / \mathrm{i}$ & $\mathrm{s} / \mathrm{i}$ & $\mathrm{s} / \mathrm{i}$ & 55,76 & 56,11 & 57,13 \\
II & $\mathrm{s} / \mathrm{i}$ & $\mathrm{s} / \mathrm{i}$ & $\mathrm{s} / \mathrm{i}$ & 58,79 & 57,35 & 55,77 \\
III & $\mathrm{s} / \mathrm{i}$ & $\mathrm{s} / \mathrm{i}$ & $\mathrm{s} / \mathrm{i}$ & 59,39 & 58,63 & 58,57 \\
\hline Total & - & - & - & 56,78 & 57,40 & 57,14 \\
\hline
\end{tabular}

Fuente: elaboración propia, en base al CONACyT (s. f.).

\section{Análisis de la Línea de Acción Sectorial 6.3.3}

En relación con la LAS 6.3.3, la distribución de Becas Nacionales adjudicadas por el CONACyT entre 2014 y 2017 reflejó valores cercanos a la paridad de género, aunque con una leve mayoría de hombres en todos los años (Tabla 13). Si el análisis se realiza por áreas del conocimiento (Tabla 14), se puede observar que la participación de mujeres fue significativamente menor en las áreas I (Física, Matemática y Ciencias de la Tierra) y VII (Ingenierías) (consideradas prioritarias por el PND13-18) y mayor en el área III (Ciencias Médicas y de la Salud). En las restantes, la distribución entre mujeres y hombres se aproximó a la paridad. 


\section{Tabla 13}

Porcentaje de Becas Nacionales adjudicadas por el CONACyT a mujeres

\begin{tabular}{lcccccc}
\hline Variable & 2013 & 2014 & 2015 & 2016 & 2017 & 2018 \\
\hline $\begin{array}{l}\text { Línea base } \\
\text { Resultado }\end{array}$ & & 47,04 & 46,42 & 47,91 & 46,88 & s/i \\
\hline
\end{tabular}

Fuente: elaboración propia, en base al CONACyT (s. f.).

\section{Tabla 14}

Porcentaje de Becas Nacionales adjudicadas por el CONACyT a mujeres, por área del conocimiento

\begin{tabular}{|c|c|c|c|c|c|c|c|c|c|c|c|c|c|c|}
\hline \multirow{3}{*}{$\begin{array}{l}\text { Área del Conocimiento del } \\
\text { CONACyT }\end{array}$} & \multicolumn{2}{|c|}{2013} & \multicolumn{2}{|c|}{2014} & \multicolumn{2}{|c|}{2015} & \multicolumn{2}{|c|}{2016} & \multicolumn{2}{|c|}{2017} & \multicolumn{2}{|c|}{2018} & \multicolumn{2}{|c|}{ Prom. } \\
\hline & $\%$ & & $\%$ & $\%$ & $\%$ & $\%$ & $\%$ & $\%$ & $\%$ & $\%$ & $\%$ & $\%$ & $\%$ & $\%$ \\
\hline & M & Á & M & Á & M & Á & M & Á & M & Á & M & Á & M & Á \\
\hline $\begin{array}{l}\text { I. Física, Matemática y Cs. de la } \\
\text { Tierra }\end{array}$ & $\mathrm{s} / \mathrm{i}$ & $\mathrm{s} / \mathrm{i}$ & 36 & 7 & 35 & 8 & 33 & 7 & 33 & 7 & $\mathrm{~s} / \mathrm{i}$ & s/i & 34 & 8 \\
\hline II. Biología y Química & $\mathrm{s} / \mathrm{i}$ & $\mathrm{s} / \mathrm{i}$ & 52 & 11 & 52 & 11 & 51 & 12 & 51 & 12 & $\mathrm{~s} / \mathrm{i}$ & $\mathrm{s} / \mathrm{i}$ & 51 & 11 \\
\hline III. Cs. Médicas y de la Salud & $\mathrm{s} / \mathrm{i}$ & s/i & 65 & 8 & 65 & 9 & 64 & 10 & 63 & 10 & $\mathrm{~s} / \mathrm{i}$ & $\mathrm{s} / \mathrm{i}$ & 64 & 9 \\
\hline $\begin{array}{l}\text { IV. Humanidades y Cs. de la } \\
\text { Conducta }\end{array}$ & $\mathrm{s} / \mathrm{i}$ & $\mathrm{s} / \mathrm{i}$ & 57 & 15 & 56 & 14 & 57 & 15 & 57 & 15 & $\mathrm{~s} / \mathrm{i}$ & s/i & 56 & 15 \\
\hline V. Cs. Sociales & $\mathrm{s} / \mathrm{i}$ & s/i & 51 & 24 & 51 & 23 & 54 & 22 & 52 & 20 & $\mathrm{~s} / \mathrm{i}$ & s/i & 52 & 22 \\
\hline $\begin{array}{l}\text { VI. Biotecnología y Cs. } \\
\text { Agropecuarias }\end{array}$ & $\mathrm{s} / \mathrm{i}$ & $\mathrm{s} / \mathrm{i}$ & 51 & 11 & 50 & 11 & 53 & 11 & 52 & 12 & $\mathrm{~s} / \mathrm{i}$ & $\mathrm{s} / \mathrm{i}$ & 51 & 11 \\
\hline VII. Ingenierías & $\mathrm{s} / \mathrm{i}$ & $\mathrm{s} / \mathrm{i}$ & 29 & 23 & 29 & 24 & 30 & 22 & 29 & 23 & $\mathrm{~s} / \mathrm{i}$ & $\mathrm{s} / \mathrm{i}$ & 29 & 23 \\
\hline Promedio & - & & 47 & & 46 & & 48 & & 47 & & - & & 47 & \\
\hline
\end{tabular}

Nota. Las columnas “\% M" representan el porcentaje de nuevas becarias en un área del conocimiento, respecto del total de becas adjudicadas en dicha área en un año determinado. En tanto, las columnas “\% Á”, representan la participación relativa de un área del conocimiento en el total de becas adjudicadas en un año específico.

Fuente: elaboración propia, en base al CONACyT (s. f.).

Por otra parte, si bien la participación de las mujeres en el SNI, con relación a la de los hombres, fue sensiblemente inferior durante todo el período (Tabla 15), pudo reconocerse un incremento leve pero sostenido, que refuerza el objetivo de la política de gobierno. Dentro del sistema, se evidenciaron algunas áreas del conocimiento en donde la participación de las mujeres es significativamente baja. Por ejemplo, en el área del conocimiento I (Física, Matemática y Ciencias 
de la Tierra), las mujeres representaron, en promedio, solo el 21\%, mientras que en el área del conocimiento VII (Ingenierías), apenas un 22\% (Tabla 16). De hecho, sólo se observó paridad en las áreas III (Ciencias Médicas y de la Salud) y IV (Humanidades y Ciencias de la Conducta), con porcentajes promedio de investigadoras del $49 \%$ y $50 \%$, respectivamente.

Tabla 15

Porcentaje de mujeres en el SNI

\begin{tabular}{lcccccc}
\hline Variable & 2013 & 2014 & 2015 & 2016 & 2017 & 2018 \\
\hline $\begin{array}{l}\text { Línea base } \\
\text { Resultado }\end{array}$ & 35,00 & & & & & \\
\hline
\end{tabular}

Fuente: elaboración propia, en base al CONACyT (s. f.).

Tabla 16

Porcentaje de mujeres en el SNI, por área del conocimiento

\begin{tabular}{|c|c|c|c|c|c|c|c|c|c|c|c|c|c|c|}
\hline \multirow{3}{*}{$\begin{array}{l}\text { Área del Conocimiento del } \\
\text { CONACyT }\end{array}$} & \multicolumn{2}{|c|}{2013} & \multicolumn{2}{|c|}{2014} & \multicolumn{2}{|c|}{2015} & \multicolumn{2}{|c|}{2016} & \multicolumn{2}{|c|}{2017} & \multicolumn{2}{|c|}{2018} & \multicolumn{2}{|c|}{ Prom. } \\
\hline & $\%$ & $\%$ & $\%$ & $\%$ & $\%$ & $\%$ & $\%$ & $\%$ & $\%$ & $\%$ & $\%$ & $\%$ & $\%$ & $\%$ \\
\hline & M & Á & M & Á & $\mathrm{M}$ & Á & M & Á & $\mathrm{M}$ & Á & $\mathrm{M}$ & Á & $\mathrm{M}$ & Á \\
\hline $\begin{array}{l}\text { I. Física, Matemática y Cs. de la } \\
\text { Tierra }\end{array}$ & 20 & 16 & $\mathrm{~s} / \mathrm{i}$ & $\mathrm{s} / \mathrm{i}$ & $\mathrm{s} / \mathrm{i}$ & $\mathrm{s} / \mathrm{i}$ & 21 & 16 & 22 & 16 & 22 & 15 & 21 & 16 \\
\hline II. Biología y Química & 42 & 17 & $\mathrm{~s} / \mathrm{i}$ & $\mathrm{s} / \mathrm{i}$ & $s / i$ & $\mathrm{~s} / \mathrm{i}$ & 42 & 16 & 42 & 16 & 42 & 15 & 42 & 16 \\
\hline III. Cs. Médicas y de la Salud & 49 & 10 & $\mathrm{~s} / \mathrm{i}$ & $\mathrm{s} / \mathrm{i}$ & $s / i$ & $\mathrm{~s} / \mathrm{i}$ & 48 & 11 & 49 & 12 & 50 & 12 & 49 & 11 \\
\hline $\begin{array}{l}\text { IV. Humanidades y Cs. de la } \\
\text { Conducta }\end{array}$ & 49 & 15 & $\mathrm{~s} / \mathrm{i}$ & $\mathrm{s} / \mathrm{i}$ & $s / i$ & $\mathrm{~s} / \mathrm{i}$ & 50 & 15 & 50 & 15 & 50 & 15 & 50 & 15 \\
\hline V. Cs. Sociales & 37 & 15 & $\mathrm{~s} / \mathrm{i}$ & $\mathrm{s} / \mathrm{i}$ & $\mathrm{s} / \mathrm{i}$ & $\mathrm{s} / \mathrm{i}$ & 38 & 16 & 39 & 16 & 40 & 16 & 39 & 16 \\
\hline $\begin{array}{l}\text { VI. Biotecnología y Cs. } \\
\text { Agropecuarias }\end{array}$ & 31 & 12 & $\mathrm{~s} / \mathrm{i}$ & $\mathrm{s} / \mathrm{i}$ & $\mathrm{s} / \mathrm{i}$ & $\mathrm{s} / \mathrm{i}$ & 34 & 11 & 35 & 12 & 36 & 12 & 34 & 12 \\
\hline VII. Ingenierías & 20 & 15 & $\mathrm{~s} / \mathrm{i}$ & $s / i$ & $s / i$ & $\mathrm{~s} / \mathrm{i}$ & 21 & 14 & 22 & 14 & 23 & 14 & 22 & 14 \\
\hline Total & 35 & & - & & - & & 36 & & 37 & & 37 & & 37 & \\
\hline
\end{tabular}

Nota. Las columnas “\% M" representan el porcentaje de nuevas investigadoras en un área del conocimiento del SNI, respecto del total de miembros del SNI en dicha área en un año determinado. En tanto, las columnas “\% A”, representan la participación relativa de un área del conocimiento en el total de miembros del SNI en un año específico.

Fuente: elaboración propia, en base al CONACyT (s. f.). 


\section{Conclusiones}

El presente trabajo se propuso evaluar el desempeño de tres líneas de acción del Objetivo Particular 3.5.2 del PECiTI14-18, orientadas a la formación y fortalecimiento del capital humano en ciencia y tecnología en México, durante el gobierno de Enrique Peña Nieto, entre 2012 y 2018. Para ello, se contrastaron indicadores oficiales y se analizaron indicadores propios.

La evaluación de la LAS 2.4.7, denominada "Incrementar el número y el nivel de los investigadores de las instituciones de educación superior en el SNI", puede dividirse en dos partes: aspectos vinculados con la cantidad y otros vinculados con la calidad. En relación con la cantidad, se evidenció un aumento en el número de investigadores por cada millón de habitantes y un incremento significativo en el número de miembros del SNI, lo que se relaciona de manera positiva con los objetivos buscados por la política pública. Sin embargo, debido a que el indicador oficial de esta acción fue corregido parcialmente, no fue posible utilizarlo como referencia para determinar el grado de satisfacción de este aspecto en relación con las expectativas; es decir, no se puede concluir si el objetivo fue finalmente alcanzado, aunque si se pueden apreciar resultados favorables.

A diferencia de la cantidad, la calidad es más difícil de observar y medir. Por un lado, como un atributo asociado a la calidad, se puede destacar el incremento en el número de artículos científicos publicados por cada millón de habitantes, cuyos resultados superaron las metas oficiales desde el año 2017. Por otro lado, se observó una caída en el porcentaje de miembros de los niveles II y III del SNI durante ese mismo período, lo que puede entenderse como un aspecto negativo, dado que el acceso a los niveles superiores exige mayores competencias por parte de los investigadores.

Por lo tanto, puede afirmarse que, sin lugar a duda, el SNI creció en cantidad durante el período 2012-2018, pero no es posible concluir que haya ocurrido lo mismo con su calidad. Para un mejor análisis en relación con esto último, resultaría pertinente profundizar en otros aspectos, tales como los resultados de las evaluaciones del SNI, el factor de impacto de las revistas en las que publican sus miembros o el número de patentes registradas por parte de los investigadores del sistema, solo por mencionar algunos ejemplos. 
Los resultados de la evaluación de la LAS 6.3.2, denominada "Incrementar el número de becarias y becarios de posgrado en ciencias e ingeniería", no fueron favorables. En primer lugar, la definición del único indicador oficial hallado y asociado a esta línea no se correspondió, exactamente, con el objetivo de la política pública, ya que midió la participación en ciencias e ingenierías de graduadas y graduados de doctorado, en lugar de becarias y becarios. De cualquier modo, los resultados del indicador fueron negativos, ya que la participación de los graduados de doctorados en esas áreas disminuyó durante todo el período.

En segundo lugar, tanto el porcentaje de nuevas Becas Nacionales adjudicadas por el CONACyT como el porcentaje de investigadores del SNI, en ciencias e ingenierías, mostraron un aumento leve a lo largo del período. Si bien este comportamiento acompaña al objetivo fijado por el gobierno, no es suficiente para afirmar que se trató del resultado de una acción deliberada por una política.

La LAS 6.3.3, denominada "Impulsar acciones afirmativas en becas de posgrados para la integración de mujeres en carreras científicas y técnicas", no pudo ser evaluada, ya que no se hallaron indicadores que definan con claridad metas anuales a contrastar. De todos modos, a través de cuatro indicadores propios, fue posible observar que la adjudicación de Becas Nacionales a mujeres por parte del CONACyT mejoró levemente su distribución por género y se aproximó a la paridad y que el porcentaje de participación de las mujeres en el SNI creció un 6,22\% en el sexenio. Sin embargo, aún con esta mejoría, su valor se ubicó, a finales de 2018, en un 37\% respecto del total de miembros.

Un aspecto llamativo son las diferencias que continúan existiendo entre la participación de mujeres y hombres en algunas áreas del conocimiento. Esta situación se refleja, fundamentalmente, en las áreas de conocimiento I (Física, Matemática y Ciencias de la Tierra) y VII (Ingenierías), consideradas prioritarias por el PND13-18. En ellas, las mujeres representaron, en promedio, solo un $34 \%$ y un $29 \%$ respectivamente de las nuevas Becas Nacionales adjudicadas por el CONACYT, y un $21 \%$ y del $22 \%$ de los miembros del SNI. Es evidente que las acciones futuras de gobierno que busquen promover la participación de las mujeres en la ciencia y la tecnología deberán enfocarse, especialmente, en ciertas áreas del conocimiento. 
En conclusión, los inconvenientes encontrados a partir de la inexistencia o las modificaciones de los indicadores oficiales dificultaron la evaluación del desempeño de las políticas públicas para la formación y fortalecimiento del capital humano en ciencia y tecnología implementadas por el gobierno del presidente Enrique Peña Nieto. Sin embargo, el análisis mediante la utilización de indicadores propios permitió evidenciar avances en algunos aspectos y retos en otros. Será pertinente revisar en el futuro si los objetivos de su sucesor, Andrés Manuel López Obrador, permitieron la continuidad de las acciones evaluadas en el presente documento y conocer si se ratificaron los logros y se corrigieron los desvíos, en pos de lograr un México más competitivo.

\section{Referencias}

(1) Barro, R. J. (1989). Economic Growth in a Cross Section of Countries. National Bureau of Economic Research, (w3120), 1-49. https://doi.org/10.3386/w3120

(2) Barro, R. J. (2001). Human Capital and Growth. American Economic Review, 91(2), 12-17. https://doi.org/10.1257/aer.91.2.12

(3) Barro, R. J. \& Sala-i-Martin, X. (1995). Economic growth. McGraw-Hill.

(4) Becker, G. S. (1964). Human capital: A theoretical and empirical analysis, with special reference to education. The University of Chicago Press.

(5) Benhabib, J. \& Spiegel, M. M. (1994). The role of human capital in economic development evidence from aggregate cross-country data. Journal of Monetary Economics, 34(2), 143173. https://doi.org/10.1016/0304-3932(94)90047-7

(6) Cabello, A. y Ortiz, E. (2013). Políticas públicas de innovación tecnológica y desarrollo: Teoría y propuesta de educación superior. Convergencia. Revista de Ciencias Sociales, 20(61), 135-172.

(7) Calderón-Villarreal, C. y Hernández-Bielma, L. (2016). Cambio estructural y desindustrialización en México. Panorama Económico, 12(23), 153-190.

(8) Cardozo, M. (2013). Políticas públicas: Los debates de su análisis y evaluación. Andamios, Revista de Investigación Social, 10(21), 39-59. https://doi.org/10.29092/uacm.v10i21.285

(9) Consejo Nacional de Ciencia y Tecnología [CONACyT]. (s. f.). Archivo Histórico del Sistema Nacional de Investigadores y Padrón de Beneficiarios. https://www.conacyt.gob.mx/ 
(10) Consejo Nacional de Ciencia y Tecnología [CONACyT]. (2014a). Programa Especial de Ciencia, Tecnología e Innovación 2014-2018. https://www.siicyt.gob.mx/index.php/normatividad/nacional/631-3-programa-especial-deciencia-tecnologia-e-innovacion-2014-2018/file

(11) Consejo Nacional de Ciencia y Tecnología [CONACyT]. (2014b). Informe general del estado de la ciencia, la tecnología y la innovación. México 2013. https://www.siicyt.gob.mx/index.php/transparencia/informes-conacyt/informe-general-delestado-de-la-ciencia-tecnologia-e-innovacion/informe-general-2013/282-informe-general2013/file

(12) Consejo Nacional de Ciencia y Tecnología [CONACyT]. (2015). Programa Especial de Ciencia, Tecnología e Innovación 2014-2018. Logros 2014. https://www.siicyt.gob.mx/index.php/transparencia/informes-conacyt/logros-programaespecial-de-ciencia-tecnologia-e-innovacion-peciti-2014-2018/1487-logros-peciti-2014/file

(13) Consejo Nacional de Ciencia y Tecnología [CONACyT]. (2016). Programa Especial de Ciencia, Tecnología e Innovación 2014-2018. Logros 2015. https://www.siicyt.gob.mx/index.php/transparencia/informes-conacyt/logros-programaespecial-de-ciencia-tecnologia-e-innovacion-peciti-2014-2018/1488-logros-peciti-2015/file

(14) Consejo Nacional de Ciencia y Tecnología [CONACyT]. (2017). Programa Especial de Ciencia, Tecnología e Innovación 2014-2018. Logros 2016. https://www.siicyt.gob.mx/index.php/transparencia/informes-conacyt/logros-programaespecial-de-ciencia-tecnologia-e-innovacion-peciti-2014-2018/3747-logros-peciti-2016/file

(15) Consejo Nacional de Ciencia y Tecnología [CONACyT]. (2018). Programa Especial de Ciencia, Tecnología e Innovación 2014-2018. Avance y Resultados 2017. https://www.siicyt.gob.mx/index.php/transparencia/informes-conacyt/logros-programaespecial-de-ciencia-tecnologia-e-innovacion-peciti-2014-2018/3904-logros-peciti-2017/file

(16) Consejo Nacional de Ciencia y Tecnología [CONACyT]. (2019a). Programa Especial de Ciencia, Tecnología e Innovación 2014-2018. Avance y Resultados 2018. https://www.siicyt.gob.mx/index.php/transparencia/informes-conacyt/logros-programaespecial-de-ciencia-tecnologia-e-innovacion-peciti-2014-2018/4824-logros-peciti-2018/file

(17) Consejo Nacional de Ciencia y Tecnología [CONACyT]. (2019b). Informe general del estado de la ciencia, la tecnología y la innovación. México 2018. 
https://www.siicyt.gob.mx/index.php/transparencia/informes-conacyt/informe-general-delestado-de-la-ciencia-tecnologia-e-innovacion/informe-general-2018/4929-informe-general2018/file

(18) Constitución Política de los Estados Unidos Mexicanos. (1917, 5 de febrero). Honorable Congreso de la Unión. Diario Oficial de la Federación.

(19) Destinobles, A. G. (2006). El capital humano en las teorías del crecimiento económico. Eumed.

(20) Fajnzylber, F. (1983). La industrialización trunca de América Latina. Centro de Economía Transnacional. Editorial Nueva Imagen.

(21) Jones, C. (1970). An Introduction to the Study of Public Policy. Wadsworth Publishing Company.

(22) Keeley, B. (2007). Human capital: How what you know shapes your life. OECD Publishing.

(23) Ley de Ciencia y Tecnología. (2002, 5 de junio). Honorable Congreso de la Unión. Diario Oficial de la Federación.

(24) Licona, Á. y Pérez, O. A. (2018). El capital humano especializado en la estrategia de ciencia y tecnología en México. Praxis Investigativa ReDIE, 10(19), 8-24. http://praxisinvestigativa.mx/revista19.html

(25) Lucas, R. E. (1988). On the mechanics of economic development. Journal of Monetary Economics, 22(1), 3-42. https://doi.org/10.1016/0304-3932(88)90168-7

(26) Majone, G. (1997). Evidencia, Argumentación y Persuasión en la Formación de Políticas Públicas. Fondo de Cultura Económica.

(27) Mankiw, N. G., Romer, D., \& Weil, D. N. (1992). A Contribution to the Empirics of Economic Growth. The Quarterly Journal of Economics, 107(2), 407-437. https://doi.org/10.2307/2118477

(28) Organización para la Cooperación y el Desarrollo Económicos [OCDE]. (2010). The High Cost of Low Educational Performance. OECD Publishing. https://www.oecd.org/pisa/44417824.pdf

(29) Poder Legislativo. (2013). Plan Nacional de Desarrollo 2013-2018, México. Diario Oficial de la Federación. http://www.dof.gob.mx/nota_detalle.php?codigo=5299465\&fecha=20/05/2013 
(30) Romer, P. (1986). Increasing Returns and Long-Run Growth. Journal of Political Economy, 94(5), 1002-1037. http://www.jstor.org/stable/1833190

(31) Romer, P. (1989). Human Capital and Growth: Theory and Evidence. National Bureau of Economic Research, (w3173), 1-49. https://doi.org/10.3386/w3173

(32) Roth, A-N. (2009). Políticas públicas. Formulación, implementación y evaluación. Ediciones Aurora.

(33) Schultz, T. W. (1959). Investment in Man: An Economist's View. Social Service Review, 33(2), 109-117. https://doi.org/10.1086/640656

(34) Vazquez, L. (2017). Revisión del modelo de sustitución de importaciones: Vigencia y algunas reconsideraciones. Economía Informa, 404, 4-17. https://doi.org/10.1016/j.ecin.2017.05.008

Cómo citar este artículo: Figueroa, D. y Basilico, N. (2021). Evaluación de políticas públicas de capital humano en ciencia y tecnología en México, 2012-2018. Tendencias, 22(2), 26-56. https://doi.org/10.22267/rtend.212202.167 


\section{Anexo A}

Indicadores utilizados para evaluar las líneas de acción del Programa Sectorial Educación del PECiTI14-18

\begin{tabular}{ll}
\hline Número & 1 \\
\hline Nombre & Porcentaje de investigadores por cada 1.000 personas de la PEA ocupada \\
\hline Fuente & PECiTI14-18 (CONACyT, 2014) \\
\hline & $I P E A_{t}=\left(\frac{N I_{t}}{P E A_{t}}\right) \times 1.000$ \\
Método de & Donde: \\
cálculo & $N I_{t}=$ Número de investigadores en el sector productivo, gobierno, educación \\
& superior e instituciones privadas sin fines de lucro en el año $t$ \\
& $P E A_{t}=$ Población económicamente activa ocupada en el año $t$ \\
\hline
\end{tabular}

\begin{tabular}{ll}
\hline Número & 2 \\
\hline Nombre & Artículos científicos publicados por cada millón de habitantes \\
\hline Fuente & PECiTI14-18 $($ CONACyT, 2014) \\
\hline & $A M H_{t}=\left(\frac{A C_{t}}{H_{t}}\right) \times 1.000 .000$ \\
Método de & Donde: \\
cálculo & $A C_{t}=$ Número de artículos científicos publicados por investigadores adscritos a \\
& $\begin{array}{l}\text { instituciones y unidades económicas mexicanas en revistas indexadas en el año } \\
\end{array}$ \\
& $H_{t}=$ Número de habitantes en el país en el año $t$ \\
\hline
\end{tabular}

\begin{tabular}{|c|c|}
\hline Número & 3 \\
\hline Nombre & Porcentaje de variación de la cantidad de investigadores del SNI \\
\hline Fuente & Elaboración propia \\
\hline $\begin{array}{l}\text { Método de } \\
\text { cálculo }\end{array}$ & $\begin{array}{l}\qquad \% \Delta Q_{t}=\left[\left(\frac{Q_{t}}{Q_{t-1}}\right)-1\right] \times 100 \\
\text { Donde: } \\
\% \Delta Q_{t}=\text { Porcentaje de variación en la cantidad de investigadores del SNI en el } \\
\text { año } t \\
Q_{t}=\text { Investigadores SNI en el año } t \\
Q_{t-1}=\text { Investigadores SNI en el año previo, } t-1\end{array}$ \\
\hline Justificación & $\begin{array}{l}\text { Debido a que parte del objeto de la LAS } 2.4 .7 \text { es incrementar la cantidad de } \\
\text { investigadores en el SNI, el indicador ayuda a estimar porcentualmente la } \\
\text { variación en el tiempo de este número, no capturado en los indicadores del } \\
\text { PECiTI14-18 }\end{array}$ \\
\hline
\end{tabular}




\begin{tabular}{|c|c|}
\hline Número & 4 \\
\hline Nombre & Porcentaje de investigadores en los niveles II y III del SNI \\
\hline Fuente & Elaboración propia \\
\hline $\begin{array}{l}\text { Método de } \\
\text { cálculo }\end{array}$ & $\begin{array}{l}\qquad \% S N I_{-} I I y I I I_{t}=\frac{\left(Q 2_{t}+Q 3_{t}\right)}{Q_{t}} \times 100 \\
\text { Donde: } \\
\% S N I_{-} I I y I I I_{t}=\% \text { de investigadores en los niveles II y III del SNI en el año } t \\
Q I I_{t}=\text { Cantidad investigadores en el nivel II del SNI en el año } t \\
Q I I I_{t}=\text { Cantidad investigadores en el nivel III del SNI en el año } t \\
Q_{t}=\text { Cantidad de investigadores en el SNI en el año } t\end{array}$ \\
\hline Justificación & $\begin{array}{l}\text { Debido a que parte del objeto de la LAS } 2.4 .7 \text { es incrementar el nivel de los } \\
\text { investigadores en el SNI, este indicador permite reconocer cuánto representa el } \\
\text { número de investigadores en niveles superiores del SNI, es decir II y III, con } \\
\text { respecto al total, no capturado en los indicadores del PECiTI14-18 }\end{array}$ \\
\hline Número & 5 \\
\hline Nombre & $\begin{array}{l}\text { Porcentaje de graduados de doctorado en ciencias e ingenierías respecto al total } \\
\text { de graduados de doctorado }\end{array}$ \\
\hline Fuente & PECiTI14-18 (CONACyT, 2014) \\
\hline $\begin{array}{l}\text { Método de } \\
\text { cálculo }\end{array}$ & $\begin{array}{l}P D C I_{t}=\left(\frac{D C I_{t}}{D T_{t}}\right) \times 100 \\
\text { Donde: } \\
D C I_{t}=\text { Graduados de doctorado en ciencias e ingeniería en el año } t \\
D T_{t}=\text { Graduados totales de doctorado en el año } t\end{array}$ \\
\hline
\end{tabular}

\begin{tabular}{ll}
\hline Número & 6 \\
\hline Nombre & Porcentaje de Becas Nacionales del CONACyT en ciencias e ingenierías \\
\hline Fuente & Elaboración propia \\
\hline & \multicolumn{1}{c}{$\% B C y T_{t}=\frac{\left(B I_{t}+B I I_{t}+B V I_{t}+B V I I_{t}\right)}{B_{t}}$} \\
& Donde: \\
& $\% B C y T_{t}=$ Porcentaje de Becas Nacionales del CONACyT en ciencias e \\
& ingenierías en el año $t$ \\
Método de & $B I_{t}=$ Becas Nacionales CONACyT en el área del conocimiento I en el año $t$ \\
cálculo & $B I I_{t}=$ Becas Nacionales CONACyT en el área del conocimiento II en el año $t$ \\
& $B V I_{t}=$ Becas Nacionales CONACyT en el área del conocimiento VI en el año $t$ \\
& $B V I I_{t}=$ Becas Nacionales CONACyT en el área del conocimiento VII en el \\
& año $t$ \\
& $B_{t}=$ Total de Becas Nacionales CONACyT en el año $t$ \\
\hline
\end{tabular}


Debido a que el objeto de la LAS 6.3.2 consiste en incrementar la cantidad de becarias y becarios de posgrado en ciencias e ingenierías, este indicador permite Justificación reconocer cuánto representa el número de nuevas Becas Nacionales otorgadas en las áreas de conocimiento de interés, es decir I, II, VI y VII, sobre el total de nuevas Becas Nacionales asignadas, no capturado en los indicadores del PECiTI14-18

\begin{tabular}{|c|c|}
\hline Número & 7 \\
\hline Nombre & Porcentaje de investigadores del SNI en ciencias e ingenierías \\
\hline Fuente & Elaboración propia \\
\hline \multirow{8}{*}{$\begin{array}{l}\text { Método de } \\
\text { cálculo }\end{array}$} & $\% Q C y T_{t}=\frac{\left(Q I_{t}+Q I I_{t}+Q V I_{t}+Q V I I_{t}\right)}{Q_{t}}$ \\
\hline & Donde: \\
\hline & $\begin{array}{l}\% Q C y T_{t}=\text { Porcentaje de investigadores del SNI en las áreas de conocimiento } \\
\text { I. II. VI y VII en el año } t\end{array}$ \\
\hline & $\begin{array}{l}Q I_{t}=\text { Cantidad de investigadores del SNI en el área del conocimiento I en el } \\
\text { año } t\end{array}$ \\
\hline & $\begin{array}{l}Q I I_{t}=\text { Cantidad de investigadores del SNI en el área del conocimiento II en el } \\
\text { año } t\end{array}$ \\
\hline & $\begin{array}{l}Q V I_{t}=\text { Cantidad de investigadores del SNI en el área del conocimiento VI en } \\
\text { el año } t\end{array}$ \\
\hline & $\begin{array}{l}Q V I I_{t}=\text { Cantidad de investigadores del SNI en el área del conocimiento VII en } \\
\text { el año } t\end{array}$ \\
\hline & $Q_{t}=$ Cantidad de investigadores del SNI en el año $t$ \\
\hline Justificación & $\begin{array}{l}\text { Si bien la LAS 6.3.2 no tiene como objeto el aumento de investigadores del } \\
\text { SNI en las áreas prioritarias, el incremento de este número puede reflejar el } \\
\text { mayor acceso a becas para posgrados en estas áreas. Por tal motivo, el } \\
\text { indicador permite identificar la participación de investigadores del SNI en las } \\
\text { áreas I, II, VI, VII, no capturado por el PECiTI14-18 }\end{array}$ \\
\hline Número & 8 \\
\hline Nombre & Porcentaje de Becas Nacionales adjudicadas por el CONACyT a mujeres \\
\hline Fuente & Elaboración propia \\
\hline $\begin{array}{l}\text { Método de } \\
\text { cálculo }\end{array}$ & $\begin{array}{l}\qquad \% N B M_{t}=\frac{N B M_{t}}{N B_{t}} \\
\qquad \begin{array}{l}\text { Donde: } \\
\% N B M_{t}=\text { Porcentaje de Becas Nacionales adjudicadas por el CONACyT a } \\
\text { mujeres en el año } t\end{array}\end{array}$ \\
\hline
\end{tabular}


$N B M_{t}=$ Cantidad de Becas Nacionales adjudicadas por el CONACyT a mujeres en el año $t$

$N B_{t}=$ Total de Becas Nacionales adjudicadas por el CONACyT en el año $t$

Debido a que el objeto de la LAS 6.3.3 consiste en impulsar la integración de la mujer en carreras científicas y técnicas a través de becas de posgrado, este

Justificación indicador refleja cuánto representa el número de nuevas Becas Nacionales otorgadas por el CONACyT a mujeres, respecto del total de nuevas Becas Nacionales adjudicadas, no capturado por el PECiTI14-18

\begin{tabular}{|c|c|}
\hline Número & 9 \\
\hline Nombre & $\begin{array}{l}\text { Porcentaje de Becas Nacionales adjudicadas por el CONACyT a mujeres, por } \\
\text { área del conocimiento }\end{array}$ \\
\hline Fuente & Elaboración propia \\
\hline $\begin{array}{l}\text { Método de } \\
\text { cálculo }\end{array}$ & $\begin{array}{l}\qquad \% N B M_{i_{t}}=\frac{N B M_{i_{t}}}{N B_{i_{t}}} \\
\text { Donde: } \\
\% N B M_{i_{t}}=\text { Porcentaje de Becas Nacionales adjudicadas por el CONACyT a } \\
\text { mujeres en el área de conocimiento } i \text { en el año } t \text {, sobre el total de Becas } \\
\text { Nacionales adjudicadas por el CONACyT en el área de conocimiento } i \text { en el } \\
\text { año } t \\
N B M_{i_{t}}=\text { Cantidad de Becas Nacionales adjudicadas por el CONACyT a } \\
\text { mujeres en el área de conocimiento } i \text { en el año } t \\
N B_{i_{t}}=\text { Cantidad de Becas Nacionales adjudicadas por el CONACyT en el área } \\
\text { de conocimiento } i \text { en el año } t\end{array}$ \\
\hline Justificación & $\begin{array}{l}\text { Debido a que el objeto de la LAS } 6.3 .3 \text { consiste en impulsar la integración de } \\
\text { la mujer en carreras científicas y técnicas a través de becas de posgrado, este } \\
\text { indicador refleja cuánto representa el número de nuevas Becas Nacionales } \\
\text { otorgadas por el CONACyT a mujeres, respecto del total de nuevas Becas } \\
\text { Nacionales adjudicadas, por áreas del conocimiento, para conocer su } \\
\text { participación en las distintas disciplinas, no capturada por el PECiTI14-18 }\end{array}$ \\
\hline Número & 10 \\
\hline Nombre & Porcentaje de mujeres en el SNI \\
\hline Fuente & Elaboración propia \\
\hline $\begin{array}{l}\text { Método de } \\
\text { cálculo }\end{array}$ & $\begin{array}{l}\qquad \% Q M_{t}=\frac{Q M_{t}}{Q_{t}} \\
\text { Donde: } \\
\% Q M_{t}=\text { Porcentaje de mujeres en el SNI en el año } t\end{array}$ \\
\hline
\end{tabular}


$Q M_{t}=$ Cantidad de mujeres en el SNI en el año $t$

$Q_{t}=$ Cantidad de investigadores en el SNI en el año $t$

Si bien la LAS 6.3.3 no se refiere al SNI, el incremento del número de mujeres

Justificación investigadoras puede reflejar el mayor acceso a becas para posgrados para

ellas. Por tal motivo, este indicador ayuda a visualizar la participación de las mujeres en el SNI, no capturada por el PECiTI14-18

\begin{tabular}{|c|c|}
\hline Número & 11 \\
\hline Nombre & Porcentaje de mujeres en el SNI por área del conocimiento \\
\hline Fuente & Elaboración propia \\
\hline $\begin{array}{l}\text { Método de } \\
\text { cálculo }\end{array}$ & $\begin{array}{l}\qquad \% Q M_{i_{t}}=\frac{Q M_{i_{t}}}{Q_{i_{t}}} \\
\text { Donde: } \\
\% Q M_{i_{t}}=\text { Porcentaje de mujeres en el área de conocimiento } i \text { del SNI en el año } \\
t \\
Q M_{i_{t}}=\text { Cantidad de mujeres en el área de conocimiento } i \text { del SNI en el año } t \\
Q_{i_{t}}=\text { Cantidad de investigadores en el área de conocimiento } i \text { del SNI en el año } \\
t\end{array}$ \\
\hline Justificación & $\begin{array}{l}\text { Si bien la LAS 6.3.3 no se refiere al SNI, el incremento del número de mujeres } \\
\text { investigadoras puede reflejar el mayor acceso a becas para posgrados para } \\
\text { ellas. Por tal motivo, este indicador ayuda a visualizar la participación de las } \\
\text { mujeres en el SNI, por área del conocimiento, para conocer las diferencias de } \\
\text { género en las distintas disciplinas, no capturadas por el PECiTI14-18 }\end{array}$ \\
\hline
\end{tabular}

